\title{
A New Mask Exposure and Analysis Facility
}

\author{
Edwin te Sligte ${ }^{\mathrm{a}}$, Norbert Koster ${ }^{\mathrm{a}}$, Alex Deutz ${ }^{\mathrm{a}}$, Wilbert Staring \\ TNO, Stieltjesweg 1, 2628 CK Delft, The Netherlands
}

\begin{abstract}
The introduction of ever higher source powers in EUV systems causes increased risks for contamination and degradation of EUV masks and pellicles. Appropriate testing can help to inventory and mitigate these risks. To this end, we propose EBL2: a laboratory EUV exposure system capable of operating at high EUV powers and intensities, and capable of exposing and analyzing EUV masks. The proposed system architecture is similar to the EBL system which has been operated jointly by TNO and Carl Zeiss SMT since 2005. EBL2 contains an EUV Beam Line, in which samples can be exposed to EUV irradiation in a controlled environment. Attached to this Beam Line is an XPS system, which can be reached from the Beam Line via an in-vacuum transfer system. This enables surface analysis of exposed masks without breaking vacuum. Automated handling with dual pods is foreseen so that exposed EUV masks will still be usable in EUV lithography tools to assess the imaging impact of the exposure. Compared to the existing system, large improvements in EUV power, intensity, reliability, and flexibility are proposed. Also, in-situ measurements by e.g. ellipsometry is foreseen for real time monitoring of the sample condition. The system shall be equipped with additional ports for EUVR or other analysis tools. This unique facility will be open for external customers and other research groups.
\end{abstract}

Keywords: EUV exposure mask pellicle XPS analysis contamination control

\section{INTRODUCTION}

For many years the successful introduction of EUV lithography has been postponed due to two major showstoppers. The first is the availability of reliable EUV sources, the second is the absence of defect free mask making and inspection infrastructure. The first topic is being addressed by Cymer (now ASML) and Gigaphoton. Both parties have programs on the development of these sources and show good progress on EUV power, reliability and up scaling, meaning that the possibility of EUV HVM production is coming nearby. The second topic is less suited to be addressed by a single party. Initiatives are taking place such as the actinic inspection tools being developed by K-T, Zeiss and Lasertec. However, the complete mask making process is not fully covered by these initiatives and many issues have to be addressed before a fully qualified reticle is available for HVM production. In particular, the possible introduction of EUV pellicles poses new and unforeseen problems about lifetime and stability of EUV optical components.

\subsection{ICCC at TNO}

TNO International Centre for Contamination Control (ICCC) is dedicated to developing the highest standards and practices in contamination control, for preventing and eliminating both particle contamination and molecular contamination. Both in current immersion and the latest innovations in EUV Lithography, TNO leads the industry in contamination control methods. As production moves towards smaller devices and larger substrates, TNO is addressing the contamination control challenges faced by both OEMs (equipment suppliers) and their supply chain as well as IDMs (device manufacturers).

TNO contributed to the development of the ASML EUV lithography tools [2]. Our work over the years includes development of:

- Cleaning technology for vacuum parts

- At wavelength EUV resist outgassing qualification

- Cleaning processes for EUVL optics

- Particle inspection and cleaning technology

- The reticle handler and reticle storage boxes for AD-tool

Photomask Technology 2014, edited by Paul W. Ackmann, Naoya Hayashi, Proc. of SPIE Vol. 9235, 92351F - @ 2014 SPIE · CCC code: 0277-786X/14/\$18 - doi: 10.1117/12.2083713 
- In-vacuum reticle and wafer clamping technology for AD-tool

- Suppression mechanism to prevent optics degradation due to resist outgassing

We are able to contribute to all of these fields due to our integrated systems engineering approach and our EUV laboratory. Its facilities include:

- EUV Beam Line (EBL) with EUV exposure and XPS analysis systems (described in more detail below)

- Rapid Nano particle scanner for particle detection on mask blanks [12],

- Ultra clean reticle handler [7],

- SEM with EDX capability,

- Outgassing test equipment,

- Plasma exposure facilities [4], and a

- Helium Ion Microscope [5].

This facility is based in the Van Leeuwenhoek Laboratory, which is shared with the Kavli Nanolab Delft of the TUDelft. This enables ICCC also to use the equipment of Kavli for further analysis or semiconductor manufacturing capabilities.

\subsection{EBL facility}

The unique EBL test facility was designed and installed at TNO, in close cooperation with Carl Zeiss. It has been operated jointly by TNO and Carl Zeiss SMT since 2005. It is shown in Figure 1. The facility contains an EUV Beam Line, in which samples can be exposed to EUV irradiation in a controlled environment. Attached to the Beam Line is an XPS system, which can be reached via an in-vacuum sample transfer system. This enables surface analysis of exposed samples without breaking vacuum. The compound instrument is used to develop and validate optics lifetime strategies for ASML EUV scanners[6]. Access to the Beam Line is exclusive to Zeiss; the XPS is open to external customers and research partners.

The beam line operates a Xe fueled EUV source, for pulsed EUV radiation in the band width between 10-20 nm. A high intensity is obtained by placing samples in the focus of a collector optic. A gas delivery system enables simulation of EUV tool vacuum conditions. A high dynamic range, differentially pumped, RGA monitors vacuum quality during exposures. In vacuum cleaning of samples is possible using hot filament generated atomic hydrogen in a load lock. The system is capable of accepting samples up to 1 inch diameter and $1 / 4$ inch thick.

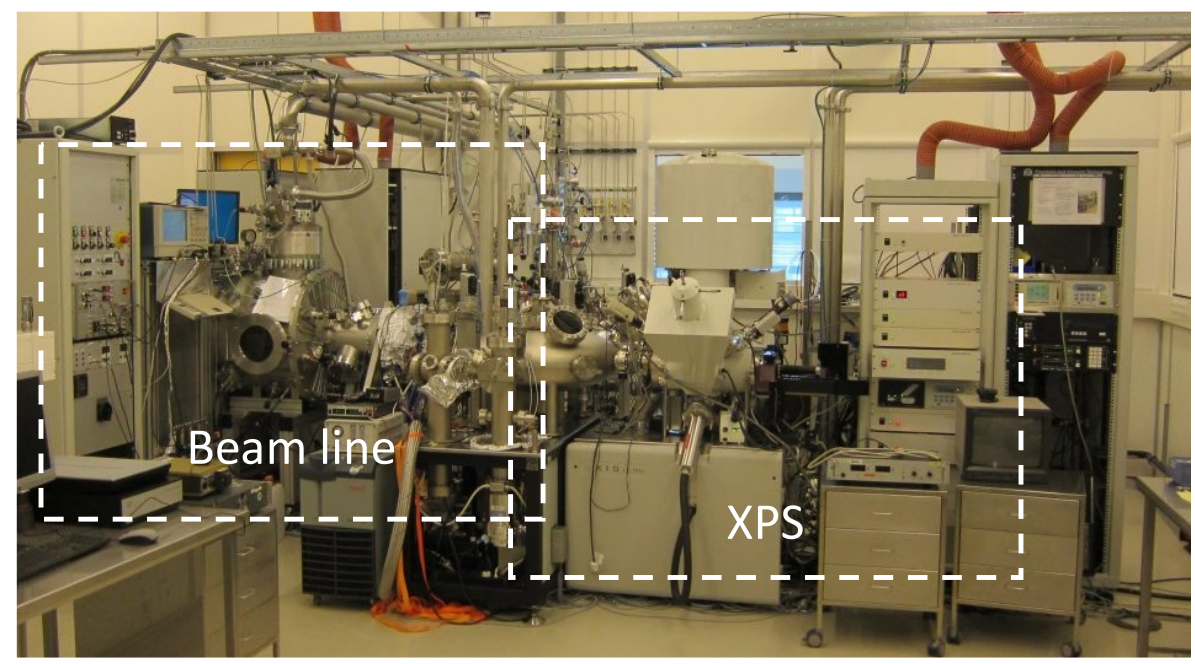

Figure 1. EBL facility at TNO 


\subsection{Motivation for a new facility}

The EUV radiation in EBL is ionizing, as it has $\sim 92 \mathrm{eV}$ of energy per photon. At high power levels and intensities, this photoionization creates a plasma[1][3]. The physics and effects of these plasma are still an open area of research. As shown in Figure 2, the current EBL is capable of generating an EUV-induced plasma. The risks associated with such a little understood phenomenon make it highly desirable to have an experimental environment with greater flexibility and higher EUV power and intensity levels.

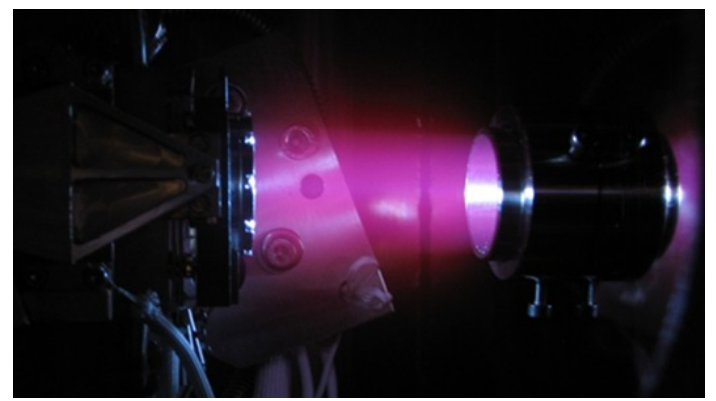

Figure 2.EUV-induced plasma at elevated gas pressure in EBL

Additionally, the current facility is not capable of handling reticles, which means that reticle lifetime testing can only be done in scanners. This is also true for testing masks with pellicles mounted. Also, XPS analysis of EUV masks is currently destructive as most XPS machines only accept smaller samples.

\section{EBL2}

The EBL2 facility is to have drastically extended functionality when compared to the present EBL. Topics on which a performance increase is to be realized include:

- EUV power and intensity capable of mimicking conditions in a $250 \mathrm{~W}$ NXE3300 scanner, and intensity scalable to future generations

- Flexibility: Samples up to and including EUV mask size including pellicle, tunable EUV spot size \& profile, and additional spectral filtering options

- Reliability: automated sample handling including a dual pod interface, improved EUV source automation, and improved dose control

- Sample analysis: In-situ ellipsometry during exposure, XPS analysis without breaking vacuum, and provision of additional ports for EUVR or other analysis tools without breaking vacuum.

EBL2 will be placed in a conditioned area to maintain compatibility with fabs and mask houses and enable shipping of clean reticles.

\subsection{System requirements}

The system is intended to match the intensity and power levels on reticles in a $250 \mathrm{~W}$ NXE3300 machine. While the detailed design of the scanner is not published, the power on such a reticle may be estimated based on public information. The throughput of the scanner at $250 \mathrm{~W}$ is 125 wafers per hour[10]. This means the scanner exposes $9 \mathrm{~m}^{2} / \mathrm{h}$ of resist. We assume $75 \%$ of the time is spent exposing resist, and the remainder is spent in wafer exchanges, alignments, etc.. At the listed dose to clear of $15 \mathrm{~mJ} / \mathrm{cm} 2$, this requires a power of $0.5 \mathrm{~W}$ hitting the wafer for a white reticle.

The POB contains 6 mirrors [11]; with the reticle included, a total of 7 reflections need to be taken into account. A white reticle has a reflectivity of over $60 \%$, but operates under small NA. A reticle at increased NA may have a $30 \%$ lower reflectivity [9] of effectively 45\%; part of the decrease is caused by increased shadowing effects. As the NA of the POB is much larger that of the reticle, but the POB mirrors are not patterned, we assume typical reflectivity for POB mirrors to be in the range from $55 \%$ to $60 \%$. We can now estimate the transmission of the POB + reticle system as 1.6 to $3 \%$. For a transmission of $2 \%$, there is $25 \mathrm{~W}$ of EUV impinging on the reticle. The illuminated area of the reticle is approximately $5 \mathrm{~mm}$ wide [8]; the length of the spot must be $4 \times 26=104 \mathrm{~mm}$ to illuminate the whole field. The resulting 
intensity is then $50 \mathrm{~mW} / \mathrm{mm}^{2}$. Given the uncertainties in the above calculation, we target an intensity of $100 \mathrm{~mW} / \mathrm{mm}^{2}$. The targeted dose control level is $20 \%$.

For maximum flexibility in defining exposures, the beam line will contain a defocusing option to tune the EUV spot size on the mask. Also, the option of installing additional optical components into the beam line to generate custom spot shapes and EUV spectra will be provided. This will enable smaller spot illumination with intensities up to 1000 $\mathrm{mW} / \mathrm{mm}^{2}$.

EBL2 will be able to accept EUV reticles, and extend the research done at the present EBL. To this end, it must accept flexible sample sizes. This is accommodated by mounting smaller samples on a custom sample holder. The gripping interfaces of the sample holder will be identical to those of EUV masks. All samples will be loaded automatically from a SEMI standard dual pod. Reticles provided in NXE compatible state should return from the facility in NXE compatible state. As a result of this design, the system will also be capable of handling masks with mounted pellicles.

Additional data on the sample will be provided by in-situ ellipsometry during exposure, enabling real-time monitoring of the condition of the exposed EUV optics. EBL2 will be able to expose samples unattended. This will enable overnight operation in our laboratory, and enable extended exposures.

Table 1. EBL2 design targets.

\begin{tabular}{|c|c|c|}
\hline Specification & EBL2 design target & EBL 1 performance \\
\hline In band EUV intensity & $100 \mathrm{~mW} / \mathrm{mm} 2$ & $6 \mathrm{~mW} / \mathrm{mm} 2$ \\
\hline Sample size & 6 inch mask & 1 inch diameter \\
\hline Total EUV power & $25 \mathrm{~W}$ & $0.7 \mathrm{~W}$ \\
\hline Uninterrupted exposure time & $>100$ hours & $3-8$ hours \\
\hline
\end{tabular}

\subsection{System architecture}

Figure 3 provides an overview of the EBL2 system architecture. The system will consist of a Transfer Unit based on ADT reticle handling equipment, with ports connecting to the various functional units. A robot will transfer masks on their inner pod base plates between the functional units attached to the Transfer Unit.

Masks are loaded from their dual pods in an atmospheric Pod Opener. An atmospheric robot will transfer the mask with its Inner Pod Base Plate into the Load Lock. After evacuating the Load Lock, the Transfer Unit can move it to other parts of the system.

As reticles are mounted face down in the Dual Pod, and need to be face up for XPS analysis, a flipping unit is provided. This unit will also provide the option to clean the mask with atomic hydrogen. Other vacuum-compatible cleaning technologies may be implemented at a later stage.

The XPS will be able to perform surface analysis on specified areas of the sample or mask surface. It will accept all sample holder assemblies that can be used in EBL2. Other types of samples may be introduced through a separate load lock.

The Beam Line Unit will consist of an EUV source, a differential pumping section containing the EUV collector, and the Exposure Chamber. The EUV source will operate on xenon. The high NA grazing incidence collector will collect and focus the EUV light on the mask or sample in the exposure chamber. The exposure chamber will have a flexible gas environment, with UHV background vacuum quality. Gases such as $\mathrm{H}_{2}, \mathrm{XCDA}$, and $\mathrm{H}_{2} \mathrm{O}$ can be added in a controlled fashion to create a customized environment for the exposure at hand. The range of partial pressures of these gases will include the relevant values for scanner emulation. Attached to the differential pumping section will be a load lock for filters and possibly EUV diagnostics. 
An additional port allows to add EUVR functionality without exposing the reticle to air.

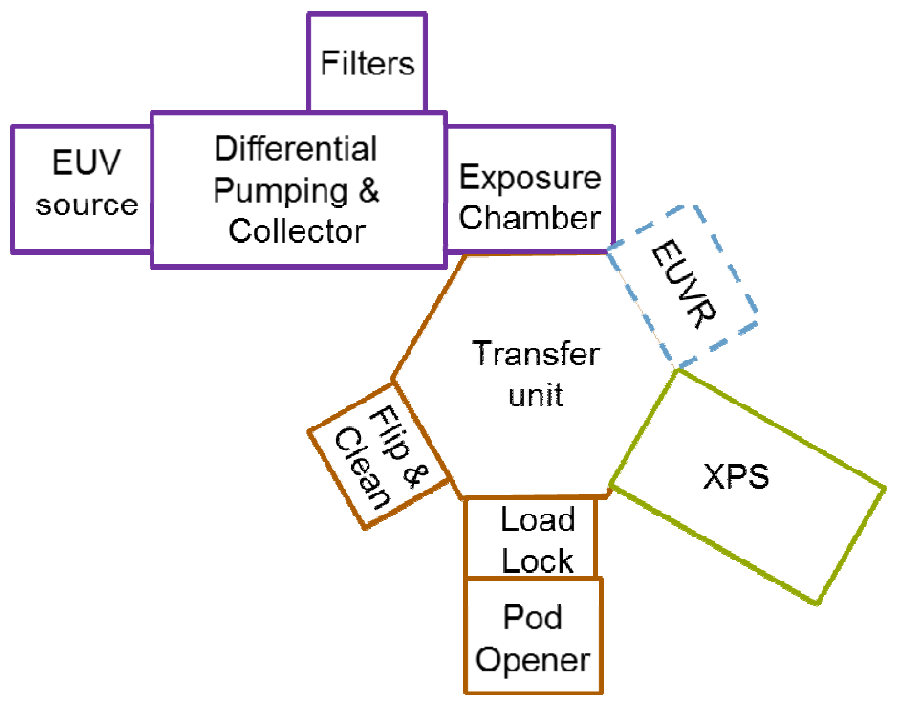

Figure 3. Architecture overview of EBL2.

\subsection{System design}

Most components of the EBL2 design are either commercially available products, or based on earlier developments at TNO. This enabled making the CAD impression in Figure 4 before starting detailed design. The hexagonal Transfer Unit that will be the pivot of the facility can be seen directly in front of the operator. Most components of the Beam Line (left) are copied from the current beam line.

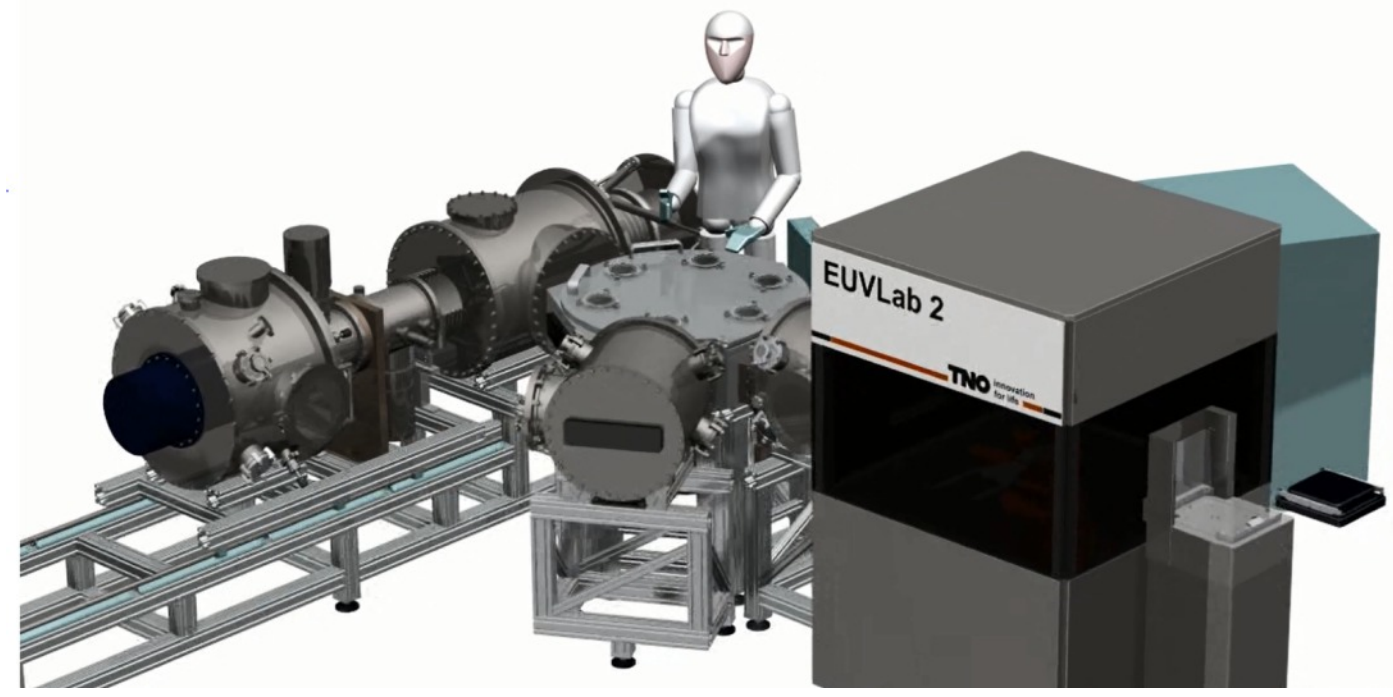

Figure 4. CAD impression of EBL2. Reticle dual pod is shown on the right.

EBL2 will be suited for characterizing and analysing phenomena such as carbon growth on EUV masks[13], oxidation of multilayer optics[14], as well as investigating the physics and effects of EUV-induced plasmas. 


\section{OUTLOOK}

The EBL2 system architecture has been baselined and detailed design is currently under way. EBL2 is planned to become operational early 2016. EBL2 will be a unique independent research facility, capable of performing both EUV exposures and XPS analyses on full reticles while maintaining NXE compatibility. In combination with our other facilities, ICCC can address optics life time, contamination control, material, pellicle and cleaning research questions.

The authors would like to thank Stefan Schmidt and Dirk Ehm of Carl Zeiss SMT GmbH for fruitful discussions. This work was made possible by the Dutch Department of Economic Affairs and Carl Zeiss SMT GmbH.

\section{REFERENCES}

[1] Proc. SPIE 8777, Damage to VUV, EUV, and X-ray Optics IV; and EUV and X-ray Optics: Synergy between Laboratory and Space III, 87771H (3 May 2013); doi: $\underline{10.1117 / 12.2018422}$

[2] http://www.sematech.org/meetings/archives/litho/8653/poster/P033_Storm_TNO.pdf

[3] Phys. Scr. T161 (2014) 014061 (5pp). doi:10.1088/0031-8949/2014/T161/0̄14061

[4] http://www.euvlitho.com/2012/S22.pdf

[5] Metrology, Inspection, and Process Control for Microlithography XXIV, edited by Christopher J. Raymond, Proc. of SPIE Vol. 7638, 763814. doi: 10.1117/12.862438

[6] N.Harned et al., EUVL Symposium, September 29 - October 2, 2008, Lake Tahoe

[7] TNO reticle handling test platform, Jacques C.J. van der Donck, Will E. Crowcombe, Erik C. Fritz, Norbert B. Koster, TNO (Netherlands). SPIE [9048-109]

[8] Martin Lowisch et al., 2012 International Symposium on Extreme Ultraviolet Lithography, Brussels

[9] Jens Timo Neumann et al., 2012 International Symposium on Extreme Ultraviolet Lithography, Brussels

[10] Extreme Ultraviolet (EUV) Lithography V, edited by Obert R. Wood II, Eric M. Panning, Proc. of SPIE Vol. 9048, 90481J. doi: 10.1117/12.2046909

[11] Extreme Ultraviolet (EUV) Lithography IV, edited by Patrick P. Naulleau, Proc. of SPIE Vol. 8679, 86791H doi: $10.1117 / 12.2012158$

[12] P. van der Walle et al. Proc. SPIE 8522, Photomask Technology 2012, 85222Q. doi:10.1117/12.2006408

[13] Y-J Fan et al., J. Vac. Sci. Technol. B 28, 321 (2010); http://dx.doi.org/10.1116/1.3333434

[14] Emerging Lithographic Technologies X, edited by Michael J. Lercel, Proc. of SPIE Vol. 6151, 615135, (2006) • 0277-786X/06/\$15 - doi: 10.1117/12.657089 